TRABAJOS DE PREHISTORIA

69, N. ${ }^{\circ}$ 2, julio-diciembre 2012, pp. 375-384, ISSN: 0082-5638

doi: $10.3989 /$ tp. 2012.12098

\title{
Ámbar en la Meseta Oriental durante el Bronce Final: yacimientos locales e importaciones bálticas
}

\author{
Amber in East Meseta during the Late Bronce Age: local sites and Baltic imports
}

\author{
M. ${ }^{a}$ Luisa Cerdeño $(*)$ \\ José Antonio Martínez (**) \\ Fernando Agua (***) \\ Teresa Sagardoy $(* * * *)$ \\ Manuel Monasterio (**)
}

\section{RESUMEN}

En el presente trabajo se dan a conocer los resultados de los análisis de espectroscopía infrarroja realizados a dos cuentas de ámbar procedentes de la fase II de la necrópolis de Herrería (Molina de Aragón, Guadalajara), fechada a finales del Bronce Final y precedente inmediato de la primera fase celtibérica de dicho cementerio. Los resultados confirman la procedencia báltica de esta materia prima. Ello la convierte en una nueva evidencia + de la llegada de elementos continentales hasta la Meseta oriental, donde dicho substrato cultural desempeñó un papel importante en la gestación de los posteriores pueblos prerromanos.

\begin{abstract}
This paper shows the results of the analysis done with two amber beads coming of the cemetery of Herreria II (Molina de Aragón, Guadalajara); it is dated in Late Bronze Age, preceding the Celtiberian period. The Baltic origin of the raw material has been confirmed by the analysis and this origin provides the evidence that continental cultural influences arrived to the meseta during mentioned period.
\end{abstract}

(*) Dpto. de Prehistoria, Facultad de Geografía e Historia, Universidad Complutense de Madrid. C/ Profesor Aranguren s/n. 28040 Madrid. Correo e.: mluisac@ghis.ucm.es

(**) Museo Comarcal de Molina de Aragón. Plaza de San Francisco s/n. 19300 Molina de Aragón.

Correos e.: j_albireo@hotmail.com; Monaste1@gmail.com

(***) Laboratorio de Arqueometría de Materiales. Centro de Ciencias Humanas y Sociales, CSIC. C/ Albasanz 26-28. 28037 Madrid. Correo e.: fernando.agua@cchs.csic.es

(****) Consejería de Educación, Cultura y Deportes, S.P. de Guadalajara. C/ Juan Bautista Topete 1-3. 19075 Guadalajara. Correo e.: tsagardoy@jccm.es

Recibido: 7-XI-2011; aceptado: 28-XII-2011.
Palabras clave: Ámbar; Península Ibérica; Siglos X-IX a.C.; Intercambios; Arqueometría.

Key words: Amber; Iberian Peninsula; Late Bronze Age; $X-I X$ BC; Changes; Archaeometry.

\section{INTRODUCCIÓN}

El ámbar ha sido una de las materias primas más apreciadas durante toda la Prehistoria europea desde el Paleolítico. Gracias a sus llamativos colores, su brillo y su facilidad para la talla ha sido ampliamente utilizado para la fabricación de variados objetos y símbolos de prestigio social. También se le ha asociado a propiedades terapéuticas, apotropaicas o sacras. Precisamente por ser una materia de fácil identificación, soporte de objetos de carácter especial y cuya procedencia se puede averiguar, su hallazgo en contextos arqueológicos la convierte en una evidencia interesante. El reconocimiento de sus fuentes de aprovisionamiento es un dato fundamental para rastrear la movilidad de determinados grupos sociales y sus posibles caminos de intercambio y comunicación.

A partir de los años 1960 se iniciaron los análisis para lograr datos fiables sobre la procedencia del ámbar. C. W. Beck (Beck et al. 1964) fue el primero en aplicar la espectrometría infrarroja para obtener series espectrográficas que mostraran los patrones característicos del mismo en cada zona. Desde entonces se realizan habitual- 
mente estas analíticas, a las que se ha incorporado también España desde hace ya una década.

Durante la Prehistoria y Protohistoria europeas el ámbar se ha obtenido mayoritariamente en la región del Báltico, aunque cada vez se conocen más ejemplos de extracción local. Recientes estudios en yacimientos de la Península Ibérica están demostrando que, en muchas ocasiones, esta materia prima es autóctona procediendo, por ejemplo, de las vetas de ámbar cretácico del norte y noreste. Los restos de ámbar local más antiguos están documentados en los niveles auriñacienses de las cuevas cántabras de Morín y El Pendo (Álvarez et al. 2005a, 2005b), en monumentos megalíticos de toda la cornisa cantábrica e incluso en yacimientos de la Edad del Bronce, como el enterramiento megalítico del Bronce Inicial de Los Lagos I (Gutiérrez 2003).

Igualmente se conocen ámbares bálticos. Posiblemente la evidencia más antigua es la del monumento megalítico de Larrarte (Guipúzcoa) de cronología calcolítica (Álvarez et al. 2005b: 175), similar a muchos enclaves portugueses desde megalitos a lugares domésticos, fechados entre el Neolítico-Calcolítico y el Bronce Final, que en general están acompañados de otras materias de prestigio como el oro, el cobre o el marfil (Vilaça et al. 2002: 76). A partir del Bronce Antiguo y Medio la mayoría de los análisis remiten al contexto nórdico. Serían ejemplo de ello yacimientos catalanes, algunos con cronologías similares a las de Herrería II, cuyos ámbares se revelaron de procedencia báltica (Rovira 1994: 79-80) o los mencionados yacimientos portugueses tardíos, como el depósito de Baiões (Vilaça et al. 2002: 65-66), fechado ya en el Bronce Tardío. El estudio de los yacimientos del noreste mostró también gran similitud con otros del sur de Francia (Hassard, Prevel, etc.) donde la procedencia báltica se constató en el $87 \%$ de las muestras analizadas (Rovira 1994: 80). Todo ello parece lógico, ya que en esta época se produjo un brillante desarrollo de las sociedades europeas y se intensificaron los contactos e intercambios entre las regiones del continente, que se prolongaron a lo largo de la Edad del Hierro.

La progresiva complejidad social y el desarrollo de grupos claramente jerarquizados dinamizó el intercambio de objetos personales, de prestigio y bienes comerciales, entre los que siempre figuraron los de ámbar, que hicieron pensar también en el desplazamiento de personas (Kristiansen y
Larsson 2006: 53). Otros autores al analizar los yacimientos del III milenio a.C. de Europa oriental sostienen también que, desde muy pronto, los movimientos de gentes facilitaron una gran distribución de diversos objetos, incluidos los de ámbar. Por ejemplo las tumbas situadas en las regiones entre el Volga y el Oká han proporcionado abundantísimos adornos de este material, interpretados como intercambios de carácter comercial o ceremonial para reforzar vínculos entre diferentes grupos (Mazurkevich 2011: 61).

Al igual que ocurre con otras materias primas cuyo lugar de obtención es restringido, la concentración es mayor cerca de sus fuentes de aprovisionamiento (Polonia, Lituania, Rusia, etc.) que en sitios más alejados. Pero esta hipótesis no siempre se cumple, ni responde a cuestiones de disponibilidad material sino a causas económicas, sociales o ideológicas. Ello podría explicar la relativa escasez de ámbar báltico en los yacimientos protohistóricos de la Península Ibérica, dada la gran distancia que les separa de los mares nórdicos y, precisamente por esto, que su presencia resulte más significativa.

Las rutas centroeuropeas de intercambio durante la Edad del Bronce están bien estudiadas. Por ellas se supone que también transitó el ámbar, aunque siempre han merecido mayor atención las que se dirigían hacia el Este de Europa, el Adriático o el Mediterráneo oriental, siendo Grecia un foco de obligada referencia (Bouzek 2007: 357). Además, autores clásicos como Plinio o Tácito citaron estos caminos hacia el Norte y la existencia de un importante comercio de esta materia durante el Imperio gracias al cual los romanos llegaron hasta aquellas regiones lejanas (Delgado 1999: 261-262). Si observamos la mayoría de los mapas al uso, la atención prestada a los territorios más occidentales es claramente menor. La Península Ibérica suele quedar excluida, pues no se sobrepasa la línea del Ródano que, enlazando con el Rin y el Elba, formó un eje por el que se accedía fácilmente hasta las tierras occidentales más lejanas (Langenheim 2003: 265). Algunos autores han señalado el marcado concepto difusionista que subyace bajo el trazado de todos estos caminos de intercambio, así como el notable actualismo de muchas de las propuestas manejadas (Palavestra 2007: 351-352).

En este contexto, consideramos de gran interés dar a conocer los resultados de los análisis de dos cuentas de ámbar recuperadas en la necrópolis de 
Herrería II (comarca de Molina de Aragón, Guadalajara), fechada a finales del Bronce Final y precedente inmediato de la primera fase celtibérica de dicho cementerio (Fig. 1). La procedencia báltica de esta materia prima creemos que es un dato fundamental para mantener la hipótesis de la llegada de las influencias continentales de los Campos de Urnas no solo hasta el extremo noreste de la Península Ibérica, sino también hasta el reborde oriental de la Meseta. Dicho substrato cultural desempeñó un papel importante en la gestación de los pueblos prerromanos allí identificados. Todo parece indicar que estas zonas meseteñas no estuvieron muy pobladas durante las primeras Edades del Bronce, quizás por su alejamiento de los circuitos culturales más activos. Eso mismo se observa en regiones cercanas, como el valle medio del Ebro que tuvo sus propios procesos culturales durante las primeras etapas metalúrgicas, no dinamizándose hasta el Bronce Final (Rodríguez de la Esperanza 2005: 181).

\section{LA NECRÓPOLIS DE HERRERÍA II (HERRERÍA, GUADALAJARA)}

La necrópolis de Herrería se excavó entre los años 1999-2005 y sus cuatro etapas de ocupación,

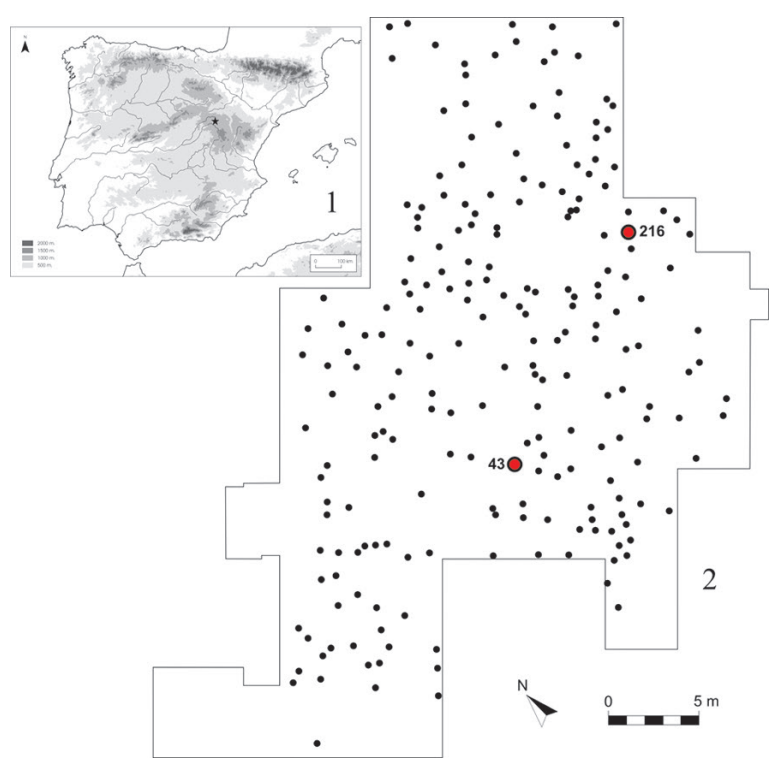

Fig. 1. 1. Localización de la necrópolis de Herrería en la Península Ibérica. 2. Distribución de las sepulturas de Herrería II, señaladas con un círculo las tumbas donde se encontraron las cuentas de ámbar. sucesivas y superpuestas, han proporcionado abundancia de datos ambientales, materiales y cronológicos que amplían sólidamente el registro arqueológico de la transición entre el Bronce Final y la Edad del Hierro en la zona (Cerdeño et al. 2002; Cerdeño y Sagardoy 2007). Este yacimiento se ha convertido en una de las claves para conocer el proceso cultural de la Meseta Oriental desde el final del II milenio a.C. hasta el momento de plenitud de la cultura celtibérica. Ya no es un caso aislado: otros enclaves están mostrando un proceso semejante, como los cercanos Fuente Estaca (Martínez 1992) o San Pedro de Oncala en Soria (Tabernero et al. 2010).

Las cuentas recuperadas en dos de las tumbas de la necrópolis protohistórica se han analizado ahora, a raíz del descubrimiento de un yacimiento de ámbar en la misma comarca. Nos parecía importante determinar si la materia prima empleada para la fabricación de estos adornos procedía de ese depósito local o de otras latitudes. Además, hasta el momento, son los únicos datos fidedignos del uso de esta materia en territorio celtibérico. La mención del marqués de Cerralbo a la existencia de fragmentos de cerámica tosca decorados con incrustaciones de círculos de “... ámbar amarillo del Báltico", procedentes de la necrópolis celtibérica de Luzaga (Aguilera y Gamboa 1916: 23) es hoy imposible de comprobar.

Como muestra la tabla 1, en la necrópolis de Herrería se identificaron claramente cuatro momentos sucesivos de ocupación protohistórica: Herrería I, II, III y IV, indicativos del uso selectivo y reiterado del lugar como enterramiento durante más de medio milenio (siglos XI-V a.C.). Sobre ellas, en el nivel más superficial, se documentó también una última fase histórica, probablemente medieval, representada por tres enterramientos y abundante material cerámico revuelto. Ello reitera la consideración funeraria y sagrada de este espacio, bastantes siglos después de las primeras utilizaciones.

En este trabajo incidimos en la fase Herrería II de la necrópolis, de donde proceden las cuentas. Se ha datado entre los siglos IX-VIII a.C. (X-IX cal. A.C.) a partir de las evidencias arqueológicas y dos fechas radiocarbónicas obtenidas sobre huesos inhumados (Cerdeño y Sagardoy 2007: 32):

GrN-25217- T57/99: $2705 \pm 55$ BP (Cal 2 sigmas BC 972-796. BP 2922-2746). 


\begin{tabular}{|c|c|c|}
\hline $\begin{array}{l}\text { HERRERÍA I } \\
\text { (66 tumbas) }\end{array}$ & $\begin{array}{l}\text { Campos de Urnas/Bronce Final } \\
\text { Autóctono } \\
\text { Siglo XI AC (XIII cal. AC) }\end{array}$ & $\begin{array}{l}\text { Incineración en hoyo } \\
\text { Señalización de estelas de piedra } \\
\text { Sin ajuar cerámico o metálico }\end{array}$ \\
\hline $\begin{array}{l}\text { HERRERÍA II } \\
\text { (229 tumbas) }\end{array}$ & $\begin{array}{c}\text { Campos de Urnas/Bronce Final } \\
\text { Autóctono } \\
\text { Siglo IX AC (X cal. AC) }\end{array}$ & $\begin{array}{l}\text { Incineración e inhumación } \\
\text { Túmulos con/sin estela central } \\
\text { Empedrados tumulares } \\
\text { Cerámicas lisas e incisas } \\
\text { Anillas y remaches de bronce } \\
\text { Cuentas de ámbar }\end{array}$ \\
\hline $\begin{array}{c}\text { HERRERÍA III } \\
\text { (153 tumbas) }\end{array}$ & $\begin{array}{l}\text { Celtibérico Antiguo } \\
\text { Siglos VII-VI AC } \\
\text { (VIII cal. AC) }\end{array}$ & $\begin{array}{l}\text { Incineración en hoyo } \\
\text { Empedrados tumulares } \\
\text { Ajuares generalizados: cerámica a } \\
\text { mano, objetos de bronce, armas de } \\
\text { hierro, cuentas collar, etc. } \\
\text { Foso delimitador con fauna } \\
\text { Cerámica a torno y a mano } \\
\text { Objetos de bronce y hierro }\end{array}$ \\
\hline $\begin{array}{c}\text { HERRERÍA V } \\
\text { (3 tumbas) }\end{array}$ & Histórica & $\begin{array}{l}3 \text { inhumaciones } \\
\text { Clavos de hierro ataúdes } \\
\text { Ajuar guerrero }\end{array}$ \\
\hline
\end{tabular}

Tab. 1. Fases culturales identificadas en la necrópolis de Herrería (Herrería, Guadalajara).

GrN-26942- T222/01: $2820 \pm 40$ BP (Cal 2 sigmas BC 1125-839; BP 2950-2700).

Destacan más de 200 enterramientos de incineración y 5 de inhumación, señalizados por variadas estructuras tumulares y objetos de ajuar como cerámicas incisas, anillas de bronce y cuentas de collar, entre ellas las dos de ámbar (Fig. 2 ), los restos óseos humanos y de fauna, carbones, etc. Las tumbas de esta fase ocupan prácticamente toda el área excavada y se distribuyen manteniendo una tendencia este-oeste. Se describen en detalle las que han proporcionado los adornos de ámbar, al parecer, sin relación especial entre sí:

Tumba 43/99: señalizada por un círculo de piedras calizas de $2 \mathrm{~m}$ de diámetro, que conservaba una hilada de altura. En el centro de la estructura, sobre una mancha de tierra carbonosa, se depositaron los restos inhumados de un neonato, huesos incinerados de un adulto y algunos objetos de ajuar. Sobre el esqueleto del neonato adherido a uno de los huesos largos, aparecieron una arandela de bronce de $3 \mathrm{~cm}$ de diámetro totalmente mineralizada y una gran cuenta de ámbar de forma tubular perforada de casi $4 \mathrm{~cm}$ de longitud y $1,7 \mathrm{~cm}$ de diámetro (Fig. 2. Tab. 1). Por debajo, había numerosos fragmentos de cerámica a mano y otros de fauna y sílex. Se identificaron dos bordes planos y rectos, uno decorado con digitaciones y otro liso. Acompañaban al primero de paredes gruesas y pasta color naranja 22 fragmentos pequeños bastante rodados, presumiblemente del mismo recipiente, y al segundo otros pequeños fragmentos de pared de cerámica gruesa, pasta color negro y superficie alisada. Además se recuperó una lasca de sílex de color beige, de tercer orden, con talón fragmentado, fractura proximal-lateral y lateral por flexión y retoque plano, marginal en la parte distal. También se recogió un incisivo y un premolar de Bos taurus y restos indeterminados de fauna.

Tumba 216/01: bajo un empedrado tumular, se conservaba una mancha de tierra carbonosa de 1,40 $\mathrm{m} \times 0,50 \mathrm{~m}$ que contenía numerosos fragmentos de carbón y huesos humanos cremados. El ajuar lo componían fragmentos dispersos de cerámica a mano: 2 de pared fina carenada, cocción oxidante y superficie alisada y 3 de cocción reductora y superficie erosionada; algunas esquirlas de bronce y una cuenta fragmentada de ámbar, cuya forma no se ha podido reconstruir.

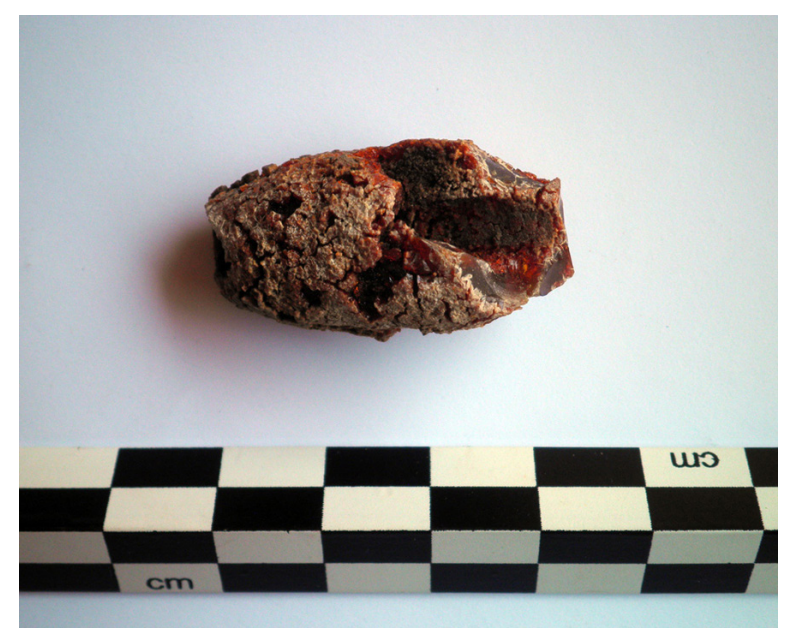

Fig. 2. Cuenta de ámbar báltico encontrada en la tumba 43/99 de la necrópolis de Herrería II. 


\section{EL ÁMBAR DE LA COMARCA MOLINESA Y EL ÁMBAR BÁLTICO}

\subsection{El yacimiento de ámbar del río Hoz Seca}

La existencia de este yacimiento era conocida desde hace tiempo por los habitantes de la zona de Peralejos de las Truchas (Guadalajara), aunque nunca hasta ahora había suscitado mayor curiosidad. El interés por las formaciones geológicas de la comarca molinesa al hilo de su posible inclusión en la 'Red Europea de Geoparques' ha motivado que el Museo Comarcal de Molina de Aragón (J. A. M. y M. M.) le haya prestado más atención por la indudable novedad que supone. Ello ha conducido a que las arqueólogas que excavaron la necrópolis de Herrería consideraran importante averiguar la procedencia de las cuentas conservadas.

El yacimiento de ámbar está en la ladera de un pequeño cañón excavado por el río $\mathrm{Hoz} \mathrm{Seca}$, afluente del río Tajo en su curso alto (Fig. 3A), que se hunde en la paramera. El relieve negativo formado puso al descubierto una serie sedimentaria del período cretácico. La serie comienza con sedimentos detríticos fluviales continentales y evoluciona hacia facies de mar abierto. Refleja los profundos cambios ambientales que ocurrieron durante este período en que se completa la fragmentación del supercontinente Pangea con la apertura de la zona norte del océano Atlántico.

En la base de la estratigrafía del cañón y constituyendo el lecho sobre el que discurre el río $\mathrm{Hoz}$ Seca, aflora un nivel formado por limos, arenas y conglomerados calcáreos en facies Weald (Fig. $3 \mathrm{~A}$, nivel 1). Corresponde a sedimentos de ambiente continental que reflejan la emersión sufrida por el terreno a causa del mínimo alcanzado por el nivel del mar de comienzos del Cretácico (Gabaldón 1989). Sobre el nivel anterior y tras una discontinuidad estratigráfica, se dispone un nivel de arenas y limos en facies Utrillas (Aguilar et al. 1971), entre los que aparecen niveles enriquecidos en carbón con las inclusiones de ámbar, objeto de nuestro interés (Fig. 3A, nivel 2 y Fig. 3B). Estos sedimentos revelan una elevación del nivel del mar con un tránsito del medio desde un ambiente fluvial, hacia facies de marisma. A continuación y en contacto concordante aparece un nivel en el que predominan materiales margosos, correspondiente al registro generado por un medio resultan-
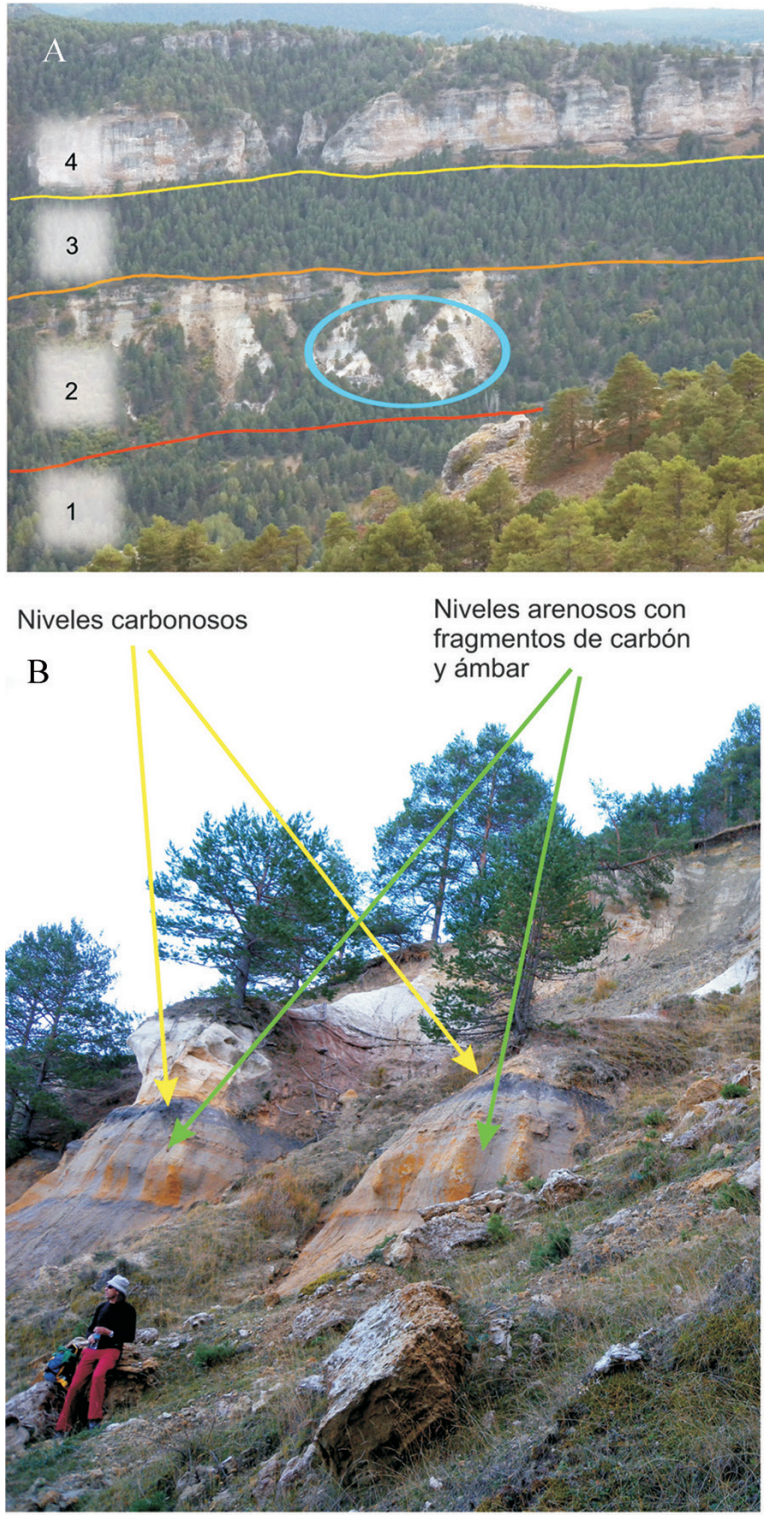

Fig. 3. A. Yacimiento de ámbar del río Hoz Seca (Peralejos de las Truchas, Guadalajara) y su contexto estratigráfico: 1. Conglomerados, areniscas y calizas en facies Weald; 2. Arenas y limos en facies Utrillas con el yacimiento de ámbar enmarcado en azul; 3. Margas de la formación Margas de Chera; 4. Calizas de la formación Dolomías de la Ciudad Encantada. B. Detalle del afloramiento de ámbar. Fotos: J. A. Martínez.

te de la evolución hacia condiciones de plataforma marina somera a causa del continuo ascenso del nivel del mar (Fig. 3A, nivel 3).

Las características paleogeográficas del yacimiento de ámbar corresponden a un ambiente de 
marisma en el que se desarrollaron frondosos bosques de proto-coníferas, sobre un sustrato húmedo poco oxigenado que favoreció la preservación en forma de carbón de la materia orgánica generada por acumulación de ejemplares muertos. El nivel se relaciona con los lignitos que se explotan económicamente en la cercana Teruel desde hace décadas. El nombre "facies Utrillas" de esta formación, procede de la localidad turolense homónima.

Desde el punto de vista sedimentológico, el yacimiento está constituido por una alternancia de arcillas caolínicas, limos y arenas multicolores con niveles intercalados enriquecidos en carbón. $\mathrm{Su}$ origen es fluvial (Pardo y Villena 1981) con influencia costera, también aparecen algunos niveles arenosos de grano fino y bien seleccionado, que revelan el desarrollo de sistemas de dunas eólicas litorales cuyos depósitos quedan incorporados a la serie (Rodríguez-López et al. 2009). En su siguiente episodio sedimentario, la progresión en la elevación del nivel del mar inundó este paisaje y generó un nuevo nivel de sedimentos, ya de carácter marino, que enterró los materiales anteriores, preservándolos de la erosión hasta la instalación de la actual red fluvial.

\subsection{El ámbar báltico}

La mayoría de los ámbares autóctonos de la Península Ibérica son del período cretácico, en general más quebradizos y por tanto más difíciles de tallar. Solo los yacimientos de algunas zonas del norte con trozos de ámbar de un tamaño suficiente se han utilizado para elaborar objetos desde tiempos muy remotos (Álvarez et al. 2005b: 176). En cambio, el ámbar báltico es de edad eocena (Cenozoico inferior) y se originó en las costas del Mar Báltico. Procede de la erosión de un estrato actualmente cubierto por el mar conocido como facies Blue Earth, resultado de la deposición de los sedimentos acarreados por un río que en aquella época atravesaba un bosque, recogiendo porciones de resina generada por sus árboles (Poinar 1992). El enterramiento de esta resina, junto con los demás materiales depositados en la desembocadura del río, desencadenaron los mecanismos de fosilización que condujeron a la generación del ámbar. Las características sedimentológicas del estrato antes mencionado son escasamente conocidas dado su carácter subacuá- tico. Estas resinas fósiles acceden flotando a la superficie, en especial tras episodios de tempestad, siendo obtenidas mediante técnicas 'pesqueras' y explotadas económicamente desde hace milenios. Su aspecto acostumbra ser más limpio y transparente, su color más claro y su textura menos quebradiza que el ámbar peninsular, por lo que es más apreciado para la elaboración de objetos de valor.

\subsection{Análisis de las muestras de Herrería II: metodología y resultados}

El estudio del ámbar con técnicas analíticas es relativamente reciente: las últimas décadas del siglo XX. Aunque su estructura es amorfa, puede considerarse un mineral orgánico $\mathrm{y}$, como tal, estudiarse mediante los métodos empleados en Geoquímica y Química orgánica. Las técnicas más utilizadas son: Espectroscopía Infrarroja (IR), Espectroscopía Infrarroja mediante Transformada de Fourier (IR-TF), Resonancia Magnética Nuclear de ${ }^{13} \mathrm{C}\left({ }^{13} \mathrm{C}-\mathrm{RMN}\right)$ y Cromatografía de Gases acoplada a Espectrometría de Masas (CG-EM) (Langheim 1969; Lambert y Frye 1982; Lambert y Poinar 2002; Beck 1997).

La Espectroscopía Infrarroja se ha utilizado ampliamente en muestras arqueológicas por su rapidez, disponibilidad y por la poca cantidad de material requerida para el análisis. Como los espectros de transmisión infrarroja de los ámbares son complejos, el método de estudio más extendido es el comparativo. Cada tipo de ámbar presenta una 'huella dactilar' característica que proporciona información geológica (antigüedad, procedencia geográfica), de su origen paleobotánico y de su estado de conservación.

Nuestro estudio se ha efectuado en un Espectrofotómetro de Infrarrojo con Transformada de Fourier (IR-TF), Perkin-Elmer modelo Spectrum 100, en el Servicio de Análisis del Instituto de Cerámica y Vidrio del CSIC. Todos los espectros se han registrado en modo transmisión en la región desde 400 hasta $4000 \mathrm{~cm}^{-1}$ de número de onda y con una resolución de $4 \mathrm{~cm}^{-1}$. Para la preparación de las muestras se ha molido en un mortero de ágata una pequeña cantidad de ámbar (1 mg aproximadamente) mezclada con $100 \mathrm{mg}$ de bromuro potásico, que es transparente a la radiación infrarroja, hasta conseguir un polvo fino y homogéneo. La mezcla se ha sometido a 
presión en una prensa hidráulica, con aplicación simultánea de vacío, hasta conseguir una pastilla en forma de disco. Como blanco se ha usado una pastilla de bromuro potásico puro, con el fin de minimizar las intensas bandas de absorción del agua residual debido al carácter higroscópico del bromuro potásico. T-216/01 se ha molido sin ningún tipo de manipulación. En cambio, T-43/99 presenta signos de alteración superficial y se ha analizado tanto la capa exterior como el interior menos alterado, para comprobar si hay diferencias en sus espectros.

Para confirmar el origen autóctono o no del ámbar arqueológico, se ha analizado una muestra de ámbar procedente del yacimiento del río $\mathrm{Hoz}$ Seca. Dista de la necrópolis unos $30 \mathrm{~km}$ y contiene trozos relativamente grandes, de tamaño centimétrico, en buen estado de conservación que podrían ser adecuados para la talla (Fig. 4).

En la figura 5.1 se representan los espectros de transmisión infrarroja de T-43/99, T-216/01 y su comparación con la muestra procedente del yacimiento del río Hoz Seca. Los espectros de las muestras de la necrópolis son muy parecidos. En ambos se observa el patrón típico de los ámbares del Báltico, una banda de absorción (disminución de la transmisión) intensa y bien definida a 1160 $\mathrm{cm}^{-1}$, junto al denominado 'hombro báltico' entre 1200 y $1250 \mathrm{~cm}^{-1}$, que corresponden a vibraciones de tensión de enlaces $\mathrm{C}-\mathrm{O}$, y una pequeña y estrecha banda situada a $885 \mathrm{~cm}^{-1}$, debida a grupos metileno $\left(=\mathrm{CH}_{2}\right)$ exocíclicos. Como estas bandas son características exclusivas de los ám-

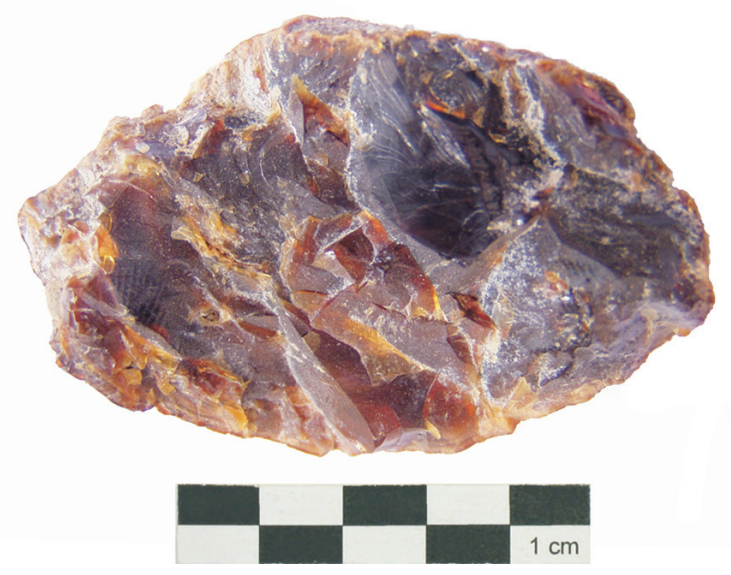

Fig. 4. Fragmento de ámbar extraído del nivel 2 del yacimiento del río $\mathrm{Hoz} \mathrm{Seca}$. bares de yacimientos terciarios bálticos y no hay depósitos geológicos de este tipo de ámbar al sur de los Alpes (Beck et al. 1965; Beck 1986; Savkevich 1981; Kosmowska-Ceranowicz 1999), podemos asegurar que las dos cuentas se fabricaron con ámbares de yacimientos situados en el norte de Europa. El espectro de la muestra de ámbar del yacimiento del río Hoz Seca difiere de los anteriores por carecer del característico 'hombro báltico' y de la banda de absorción alrededor de $890 \mathrm{~cm}^{-1}$. Además presenta unas bandas más intensas y complejas en la región entre 950 y 1200 $\mathrm{cm}^{-1}$. El espectro es similar a otros de ámbares cretácicos de la Península Ibérica, como los de Peñacerrada en Álava (Alonso et al. 2000) o Reocín en Cantabria (Matuszewska y Karwowski 1999; Matuszewska et al. 2001). La ausencia de la banda de $890 \mathrm{~cm}^{-1}$, junto con una disminución de intensidad de la banda a $1700 \mathrm{~cm}^{-1}$, que corresponde a vibraciones de tensión de enlaces $\mathrm{C}=\mathrm{O}$, es indicativa de una mayor madurez del ámbar (Grimalt et al. 1998), lo que concordaría con su mayor antigüedad, ya que se formó durante el período cretácico, hace unos 100 millones de años.

El ámbar es un material que se degrada con facilidad con el aire por los procesos de oxidación que sufren sus componentes. En la figura 5.2 se representan los espectros del interior de T43/99 y de la capa externa alterada. En este último tanto el hombro báltico entre 1200 y 1250 $\mathrm{cm}^{-1}$, como la banda de $885 \mathrm{~cm}^{-1}$, han disminuido mucho en intensidad. Estos efectos confirman su estado de deterioro (Stout et al. 2000; Sashoua 2002 (1); Sashoua et al. 2005).

\section{CONSIDERACIONES FINALES}

La transición entre el II-I milenio a.C. en el sector oriental de la Meseta creemos que fue un momento clave durante el que se desarrollaron cambios profundos en las sociedades que allí habitaron. A través de las evidencias arqueológicas se detecta una creciente complejidad social y tecnológica manifestada en la ocupación de nuevas tierras, un evidente aumento demográfico, nuevos ritos, nuevas formas de organizar el espacio fune-

(1) Citado en Pastorelli, G., 2009: Archaeological Baltic amber: degradation, mechanisms and conservation measures. Tesis Doctoral. Universidad de Bolonia. 

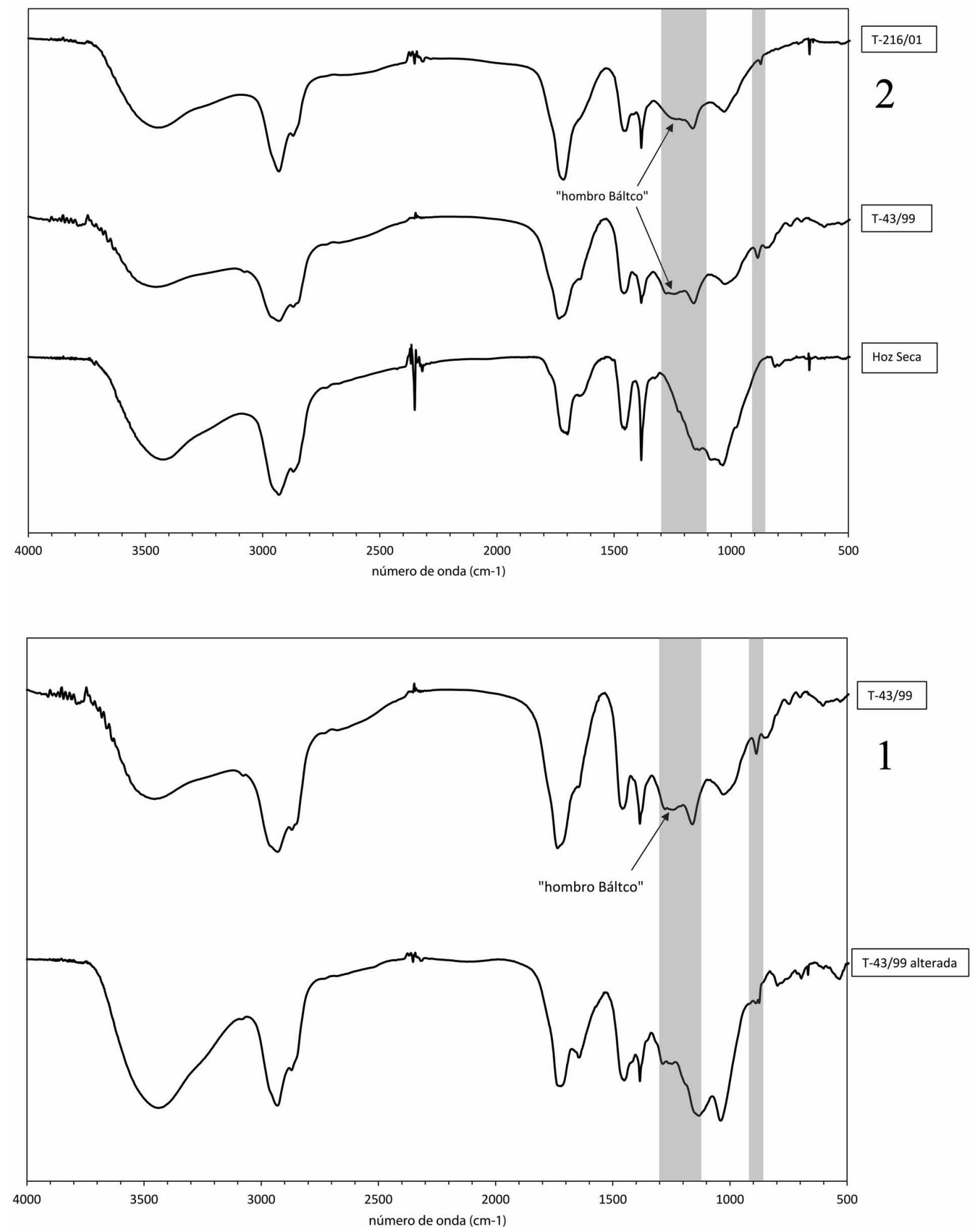

Fig. 5. 1. Espectros de transmisión infrarroja de las muestras, del interior y de la capa externa alterada, de Herrería y del yacimiento del río Hoz Seca. 2. Espectros de transmisión infrarroja de las muestras T-43/99, alterada y sin alterar, de la necrópolis de Herrería II (Molina de Aragón, Guadalajara) y del yacimiento del río Hoz Seca. 
rario y nuevos elementos materiales. Esta época tiene el interés añadido de ser el precedente inmediato de la cultura celtibérica, a la que se reconoce perfectamente un siglo y medio después y que mantuvo a lo largo de toda la Edad del Hierro muchos de los rasgos culturales que entonces se perfilaron, especialmente en el ámbito funerario según se constata, por ejemplo, en la secuencia ininterrumpida de la necrópolis de Herrería.

La presencia de cuentas de ámbar en ajuares molineses de los siglos IX-VIII a.C. es en sí misma importante, al identificarse por primera vez el uso de este material en la región. Su lejana procedencia aporta nuevos datos para la interpretación de sus fases culturales. Su origen alóctono es muy elocuente. Sugiere que, aunque había un yacimiento local perfectamente accesible a menos de una jornada de distancia a pie, o no se conocía esta resina fósil o los anteriores grupos asentados en la comarca no le dieron un significado relevante, al contrario de lo que ocurría en otros ámbitos peninsulares y en casi toda Europa continental, donde sí fue más frecuente la utilización de objetos de ámbar.

Los resultados de nuestros análisis, demostrando que el ámbar empleado en la fabricación de las cuentas de Herrería II procede del Báltico, hacen pensar en la llegada de gentes con nuevos presupuestos sociales, que sí conocían y valoraban este elemento y/o en el establecimiento de contactos o intercambios por los que llegó esta materia prima junto a otros materiales e ideas que se identifican en el cementerio. Entre las novedades culturales subrayamos sobre todo el ritual incinerador y la ubicación y forma del cementerio. Consideramos que se deben a influencias llegadas hasta estas tierras desde el medio Ebro y hasta allí, a su vez, del otro lado de los Pirineos y a las que otorgamos un papel importante en la gestación de los posteriores pueblos prerromanos.

Este trabajo es un ejemplo más de la necesidad que tiene la investigación arqueológica de elaborar un registro material sólido. Ello se consigue en muchas ocasiones acudiendo a técnicas especializadas aplicadas por colegas de otras disciplinas, es decir, a la Arqueometría, un campo interdisciplinar entre las ciencias naturales y las ciencias humanas, capaz de proporcionar nuevas pruebas empíricas que permiten mantener hipótesis interpretativas como la esbozada en las líneas precedentes.

\section{AGRADECIMIENTOS}

Este trabajo se ha realizado dentro del Proyecto I+D, Ref: HAR2010-21976. Laura Peláez del Servicio de Análisis del Instituto de Cerámica y Vidrio (CSIC) efectuó los espectros de transmisión infrarroja.

\section{BIBLIOGRAFÍA}

Aguilar, M. J.; Ramírez, J. y Riba, O. 1971: “Algunas precisiones sobre la sedimentación y paleontología del Cretácico inferior en la zona de Utrillas-Villarroya de los Pinares". Estudios Geológicos 27: 497-512.

Aguilera y Gamboa, E. 1916: Las necrópolis ibéricas. Asociación Española para el Progreso de las Ciencias. Madrid.

Alonso, J.; Arillo, A.; Barrón, E.; Corral, J. C.; Grimalt, J.; López, J. F.; López, R.; Martínez-Delclós, X.; Ortuño, V.; Peñalver, E. y Trincao, P. R. 2000: "A new fossil resin with biological inclusions in Lower Cretaceous deposits from Alava (Northern Spain, Basque-Cantabrian Basin)". Journal of Paleontology 74 (1): 158-178.

Álvarez, E.; Peñalver, E. y Delclòs, X. 2005a: "Presencia de ámbar local en los niveles auriñacienses de Cueva Morín y El Pendo (Cantabria, España)". En J. A. Lasheras y R. Montes (eds): Neandertales cantábricos. Estado de la cuestión. Monografías del Museo Nacional y Centro de Investigación de Altamira 22, Ministerio de Cultura. Madrid: 10-38.

Álvarez, E.; Peñalver, E. y Delclós, X. 2005b: "La presencia de ámbar en los yacimientos prehistóricos (del Paleolítico superior a la Edad del Bronce) de la Cornisa Cantábrica y sus fuentes de aprovisionamiento". Zephyrus 58: 159-182.

Beck, C. W. 1986: "Spectroscopic investigations on amber". Applied Spectroscopy Reviews 22: 57-110.

Beck, C. W. 1997: "Détermination de la provenance des resines fossiles par l'analyse spectrale en infrarouge". En D. Leesch: Hauterive-Champréveyres. Un campement magdalénien au bord du lac de Neuchâtel. Cadre chronologique et culturel mobilier et structures, analyse spatiele (Sector 1). Archéologie neuchâteloise 19. Neuchâtel: 105-107.

Beck, C. W.; Wilbur, E. y Meret, S. 1964: "Infrared spectra and the origin of amber". Nature 201: 256-25.

Beck C. W.; Wilbur E.; Meret S.; Kossove D. y Kermani K. 1965: "The infrared spectra of amber and the identification of Baltic amber". Archaeometry 8: 96-109.

Bouzek, J. 2007: "The amber route, Apollo and the Hyperboreans". En I. Galanaki, H. Tomas, Y. Galanakis y R. Laffineur (eds.): Between Aegean and 
Baltic seas. Prehistory across borders. Proceedings of the International Conference (Zagreb, 2005). Aegaeum 27: 357-362.

Cerdeño, M. ${ }^{a}$ L.; Marcos, F. y Sagardoy, T. 2002: "Campos de Urnas en la Meseta Oriental: nuevos datos sobre un viejo tema". Trabajos de Prehistoria 59 (2): 132-147.

Cerdeño, M. ${ }^{a}$ L. y Sagardoy, T. 2007: La necrópolis celtibérica de Herrería III y IV. Estudios Celtibéricos de Segeda 3. Zaragoza.

Delgado, J. A. 1999: La gran ruta del ámbar en la época imperial romana: vía de intercambio comercial y contacto cultural. Diputación Foral de Álava. Vitoria.

Gabaldón, V. 1989: Mapa Geológico de España 1:50000. Hoja 539 Peralejos de las Truchas, Instituto Tecnológico GeoMinero de España. Madrid.

Grimalt J. O.; Simoneit, B. R. T.; Hatcher P. G. y Nissenbaum A. 1988: "The molecular composition of ambers". Organic Geochemistry 13: 677-690.

Gutiérrez, A. 2003: "Sustancias exóticas en la Prehistoria reciente del sur de Cantabria: el ámbar de Los Lagos I (Campoo de Suso)". C.A.E.A.P., 25 años de Investigaciones sobre el Patrimonio Cultural de Cantabria. Ayuntamiento de Camargo. Santander: 137-144.

Kosmowska-Ceranowicz, B. 1999: "Succinite and some other fossil resins in Polandand Europe (deposits, finds, features and differences in 1RS)". Estudios del Museo de Ciencias Naturales de Álava 14 (n. ${ }^{\circ}$ especial 2): 73-117.

Kristiansen, K. y Larsson, T. B. 2006: La emergencia de las sociedades del Bronce. Viajes, transmisiones $y$ trasformaciones. Bellaterra-Arqueología. Barcelona.

Lambert, J. B. y Frye, J. S. 1982: “Carbon Functionalities in Amber". Science 217: 55-57.

Lambert, J. B. y Poinar G. O. Jr. 2002: "Amber the organic gemstone". Accounts Chemical Reserches 35: 628.

Langenheim, J. H. 1969: “Amber: a botanical inquiry”. Science (163) 3872: 1157-1169.

Langenheim, J. H. 2003. Plant resins: Chemistry, Evolution, Ecology, and Ethnobotany. Timber Press. Portland, Oregon.

Martínez, V. 1992: "El poblado de Campos de Urnas de Fuente Estaca (Embid, Guadalajara)". En J. Valiente (ed): La celtización del Tajo Superior. Universidad de Alcalá de Henares. Alcalá de Henares: 67-78.

Matuszewska, A. y Karwowski, L. 1999: "Physicochemical analysis of the molecular and macromolecular phases of Baltic amber". Estudios del Museo de Ciencias Naturales de Álava 14 (n. ${ }^{\circ}$ especial 2): 49-62.

Matuszewska, Á.; Wrzalik, R. y Hacura, A. 2001: "Reflection micro-FT IR spectroscopy of fossil re- sins and synthetic polymers". Prace Muzeum Ziemi 46: 67-75.

Mazurkevich, A. N. 2011: "Del Neolítico al Bronce antiguo en el área forestal de Europa Oriental (VII milenios a. C.)". En J. A. López Padilla (ed.): Ermitage. Tesoros de la arqueología rusa en el $M A R Q$. Fundación MARQ-Museo Arqueológico de Alicante. Alicante: 53-65.

Palavestra, A. 2007: "Where there an Amber Route?". En I. Galanaki, H. Tomas, Y. Galanakis y R. Laffineur (eds.): Between Aegean and Baltic seas. Prehistory across borders. Proceedings of the International Conference (Zagreb, 2005). Aegaeum 27: 349-356.

Pardo, G. y Villena, J. 1981: “Geología de la Región de Montalbán-Utrillas". En M. Gutiérrez (ed.): XV Curso de Geología Práctica: 161-194. Teruel.

Poinar, G. O. 1992: Life in amber. Stanford University Press. Stanford.

Rodríguez de la Esperanza, M. J. 2005: Metalurgia y metalúrgicos del valle del Ebro. Bibliotheca Archaeologica Hispana 24, Real Academia de la Historia. Madrid.

Rodríguez-López, J. P.; Meléndez, N.; Soria, A. R. y Boer, P. L. 2009: "Reinterpretación estratigráfica y sedimentológica de las Formaciones Escucha y Utrillas de la Cordillera Ibérica". Revista de la Sociedad Geológica de España 22: 163-215.

Rovira, J. 1994: “Ámbar y pasta vítrea. Elementos de prestigio entre el neolítico avanzado y el bronce final en el noreste de la Península Ibérica. Un primer estado de la cuestión". Quaderns de Prehistoria i Arqueologia de Castelló 16: 67-92.

Sashoua, Y. 2002: "Degradation and inhibitive conservation of Baltic amber in collections". Report for Culture Minister and National Museum of Denmark.

Sashoua, Y.; Degn Berthelsen, M. L. y Nielsen, O. F. 2005: "Raman and ATR-FTIR spectroscopies applied to the conservation of archaeological Baltic amber". Journal of Raman Spectroscopy 37: 1221-1227.

Savkevich, S. S. 1981: "Physical Methods Used to Determine the Geological Origin of Amber and Other Fossil Resins; Some Critical Remarks". Physical Chemistry Minerals 7: 1-4.

Stout, E. C.; Beck, C. W. y Anderson, K. B. 2000: "Identification of Rumanite (Romanian amber) as thermally altered succinite (Baltic amber)". Physics and Chemistry of Minerals 27: 665-678.

Tabernero, C.; Sanz, A. y Benito, J. P. 2010: "El registro funerario celtibérico en el norte de Soria". En F. Burillo (ed.): VI Simposio sobre Celtiberos: Ritos y Mitos (Daroca 2008): 391-402. Zaragoza.

Vilaça, R.; Beck, C. W. y Stout, E. C. 2002: "Provenience analysis of prehistoric amber artefacts in Portugal". Madrider Mitteilungen 43: 63-78. 